\title{
Pelvic Static Magnetic Stimulation to Control Urinary Incontinence in Older Women: A Randomized Controlled Trial
}

\author{
Marianne C. Wallis, RN, PhD; Elizabeth A. Davies, RN, PhD; Lukman Thalib, PhD; and \\ Susan Griffiths, BA
}

\begin{abstract}
Objectives: To determine the efficacy of non-invasive static magnetic stimulation (SMS) of the pelvic floor compared to placebo in the treatment of women aged 60 years and over with urinary incontinence for 6 months or more.
\end{abstract}

Subjects and Methods: A single-blinded randomized, placebo-controlled, parallel-group trial. Subjects were excluded if they had an implanted electronic device, had experienced a symptomatic urinary tract infection, or had commenced pharmacotherapy for the same in the previous 4 weeks, or if they were booked for pelvic floor or gynecological surgery within the next 3 months. Once written consent was obtained, subjects were randomly assigned to the active SMS group $(n=50)$ or the placebo group $(n=5 I)$. Treatment was an undergarment incorporating 15 static magnets of 800-1200 Gauss anterior, posterior, and inferior to the pelvis for at least 12 hours a day for 3 months. Placebo was the same protocol with inert metal disks replacing the magnets. Primary outcome measure was cessation of incontinence as measured by a 24-hour pad test. Secondary outcomes were frequency and severity of symptoms as measured by the Bristol Female Lower Urinary Tract Symptoms questionnaire (BFLUTS-SF), the Incontinence Severity Index, a Bothersomeness Visual Analog scale, and a 24-hour bladder diary. Data were collected at baseline and 12 weeks later.

Results: There were no statistically significant differences between groups in any of the outcome measures from baseline to 12 weeks. Initial evidence of subjective improvement in the treatment group compared to the placebo group was not sustained with sensitivity analysis.

Conclusion: This study found no evidence that static magnets cure or decrease the symptoms of urinary incontinence. Additional work into the basic physics of the product and garment design is recommended prior to further clinical trials research.

Keywords: Randomized controlled trial; Static magnetic therapy; Urinary incontinence

$\mathrm{U}$ rinary incontinence (UI) is defined as the "complaint of involuntary loss of urine." It affects millions of people worldwide, ${ }^{2,3}$ and has a major impact on physical well-being, quality of life, and emotional and social health. ${ }^{4-6}$ For some people with UI, medications and surgery can successfully treat the condition; however, for many people these treatments are either ineffective or only partially effective,

Corresponding Author: Professor Marianne Wallis, RN, PhD; Griffith University Research Centre for Clinical Practice Innovation; Gold Coast Campus; Queensland 4222, Australia; Email: M.Wallis@griffith.edu.au

Financial Disclosure: This study was funded by the Australian Department of Health and Ageing, National Continence Management Strategy. Australian Clinical Trials Registration Number: ACTRN I 2605000755639.

and other continence management strategies, such as containment, are required. ${ }^{7}$

Conservative or non-invasive continence management strategies are likely to be more acceptable to older women. ${ }^{8,9}$ It is important, however, to determine whether these noninvasive treatments are effective. ${ }^{10}$ Conservative management

Received: March 26, 2011

Revised: July 29, 20II

Accepted: July 31,2011

doi: $10.3121 / \mathrm{cmr} .2011 .1008$
Conflict of Interest Statement:

The authors declare no commercial conflict of interest. 

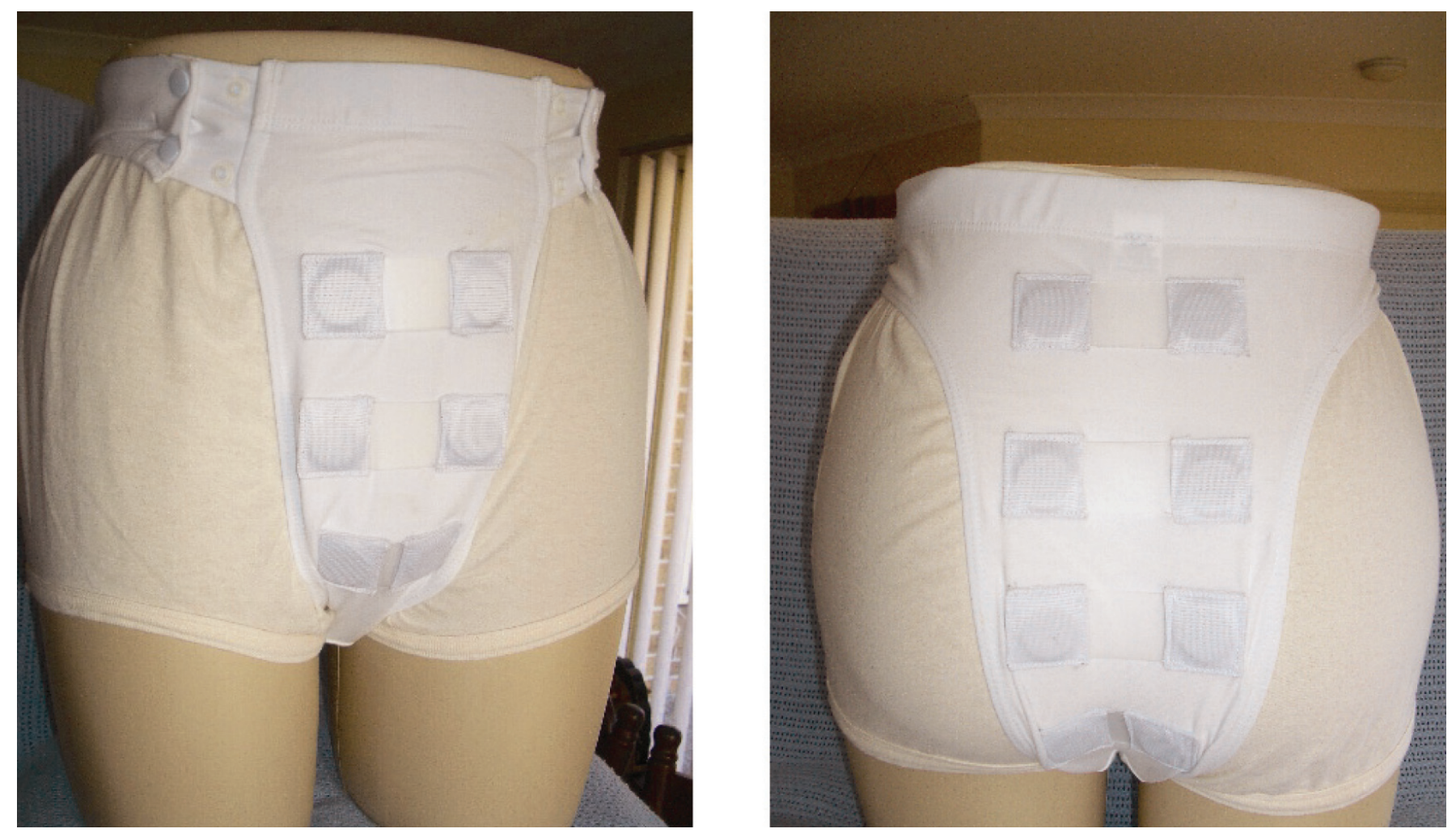

Figure 1. Photograph of magnetic pants, front and rear.

of female UI has included extracorporeal electromagnetic innervation (ExMI), for many years. In ExMI, a pulsed magnetic field is generated which induces an electrical depolarization of the nerves within the pelvic floor, consequently causing the pelvic floor muscles to contract. ${ }^{11}$ It is postulated that ExMI passes uninterrupted through clothing and penetrates all body tissues without alteration. A series of small studies incorporating a variety of research designs undertaken in the USA, ${ }^{11-12}$ Japan, ${ }^{13-14}$ South Korea, ${ }^{15}$ Australia, ${ }^{16}$ and New Zealand ${ }^{17}$ has established the acceptability of ExMI to patients. These early studies provided beginning evidence that this therapy was effective in reducing the number of incontinence and leakage episodes in women with both stress incontinence ${ }^{11}$ and overactive bladder, ${ }^{9,15}$ thus improving their quality of life. ${ }^{9,17}$ In addition, continuous electromagnetic stimulation has been shown to affect urethral closure and bladder inhibition, ${ }^{14}$ elevate urethral closure pressure, and increase bladder capacity ${ }^{13}$. However, later studies have only found a significant treatment effect in participants with poor pelvic floor tone at baseline, ${ }^{19}$ while others have found very high rates of recurrence of the incontinence. ${ }^{16,19-20}$

In terms of cost, ExMI can be quite expensive; in contrast, relatively cheap devices that incorporate static magnets are being marketed as being of potential benefit to sufferers of urinary incontinence. The World Health Organization states that available evidence does not indicate any adverse effects on human health from exposure to static magnetic fields up to 2.0 Tesla,${ }^{21}$ and animal studies have suggested that exposure to a 0.25 Tesla static magnetic field results in increased local blood flow. ${ }^{22}$ There is one study that has shown changes to skin blood perfusion with the application of static magnets. However, a recent systematic review of randomized controlled trials found that "the evidence does not support the use of static magnets for pain relief." ${ }^{\prime 23}$ Another review found that there was a need to develop static magnet dosing parameters for individual clinical conditions. ${ }^{24}$ Despite this, claims have been made about the efficacy of static magnets in the treatment of pain ${ }^{11}$ and other conditions such as incontinence, ${ }^{25}$ which are yet to be rigorously tested.

Research into the effects of static magnetic fields on the pelvic floor include one study of a magnetic shoe cushion which found that a static magnetic field increased the efficacy of voluntary levator ani contractions. ${ }^{26}$ However, there have been no studies indicating a positive effect for static magnets in the treatment of urinary incontinence. Despite this lack of evidence, there are products on the market targeting this vulnerable group of older people. The aim of our study was to estimate the efficacy and safety of a commercially available undergarment that incorporates 15 static magnets of approximately 900 Gauss each, arranged anterior, posterior, and inferior to the pelvis, compared to placebo in the treatment of older women with UI.

\section{Subjects and Methods}

A randomized, single-blind, placebo-controlled, parallelgroup study of the efficacy of static magnetic therapy in 101 women with UI was conducted. The study was funded by the 
Australian Department of Health and Ageing and ethical approval was granted by all universities and health services involved (six in total). All subjects were aged 60 years or over, were self-managing at home, able to read and write in English, and had experienced stress, urge, or mixed urinary incontinence at least once a week for the last 6 months. Subjects were excluded from the study if they had any implanted electronic device (eg, pacemaker), if they had experienced a symptomatic urinary tract infection or had commenced pharmacotherapy for the same in the previous 4 weeks, or if they were scheduled for pelvic floor or gynecological surgery within the next 3 months. In order to blind the subjects to group allocation, they were informed that the trial was assessing the effects of metals with different properties. If during the consent process the subjects insisted on knowing the specific properties of the metals, they were excluded from the trial.

The subjects were recruited from 2004 to 2005 via nurse-led incontinence clinics as well as a research-specific clinic in South East Queensland, Australia. Newspaper advertisements were placed in the catchment area. Once assessed for eligibility by the Continence Nurse Advisors (CNAs) or research assistants, participants were given information about the trial, informed consent was obtained, they were measured for the undergarment, instructed about the wearing of the undergarment, and data collection procedures were explained. Once recruited, participants' contact details were provided to an independent research assistant who randomized participants (by using a coin toss), and they were sent two sets of either the intervention or placebo garments in the correct size.

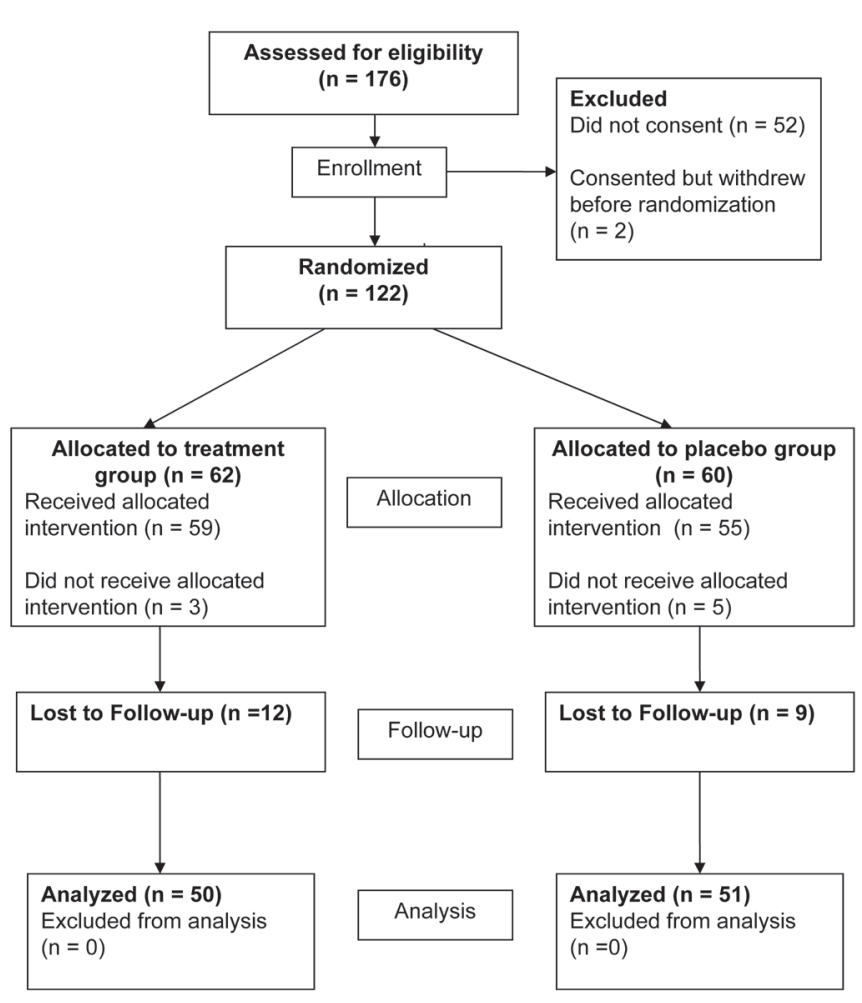

Figure 2. Clinical trial flowchart.
Power analysis using Cohen's ${ }^{27}$ tables and estimating an effect size of $50 \%$ for the primary end-point of cessation of urinary incontinence, suggested that for an alpha of 0.05 , a sample size of 53 would give a power of 0.80 . The effect size was based on data from the manufacturer. Self-reports from 100 customers indicated that over $50 \%$ had ceased to have incontinence episodes after 12 weeks of garment use. There were no other studies of a similar intervention on which sample size estimation could be based.

Data were collected at randomization and 12 weeks after randomization. At baseline the subjects completed a demographic and medical history form. They were also given a "Wearer's Diary" to complete for the 3 months of the study to assess compliance with study protocol and to collect data about product comfort. The primary outcome measure was cessation of incontinence as measured by a 24-hour Pad Test. $^{28}$ In addition, data were collected for the following secondary outcome measures: a 48-hour Bladder Diary, ${ }^{29}$ the Bristol Female Lower Urinary Tract Symptoms questionnaire (BFLUTS-SF) ${ }^{30}$ the Incontinence Severity Index (ISI), ${ }^{31}$ and a Bothersomeness visual analog scale (VAS). ${ }^{32}$ At the end of data collection all participants were telephoned and asked whether they subjectively felt there had been an improvement in their urinary incontinence. This question was asked to provide data that might explain any differences between the effect size obtained from the manufacturer's data and the final results of the trial.

The device used was a cotton and polyester undergarment, shaped like a loincloth with an adjustable waistband. Fifteen magnetic disks of 800 to 1200 Gauss each were sewn into cotton bands on the outside of the undergarment (figure 1). It is unclear whether the garment could be considered a magnetic flux generator since no information is available related to the magnetic field produced by the garment. In addition, the manufacturer makes no statement about decay of field force, and there is no indication regarding a time limit being applied to the strength of the magnetic field. A search of the literature revealed no animal studies related to this or any similar device. The placebo undergarment was the same design, but the magnets were replaced with inert metal disks of the same size and weight.

On the advice from the manufacturer, subjects were asked to wear the undergarment for a minimum of 6 consecutive hours during the day and at least 6 hours overnight. They were asked to keep a diary of exactly how many hours per day that they wore the garment and to record comments about garment use and wearability.

Data were entered and analyzed using the Statistical Package for the Social Sciences (SPSS) version 12. The demographic and outcome data were checked for normality of distribution, and most variables were found to not be normally distributed. The Mann Whitney $U$ test and the chi square test, as appropriate, were used to test the hypotheses related to the 
Table 1. Comparison of sample characteristics and medical history for intervention and control groups with descriptive statistics for total sample and groups.

\begin{tabular}{|c|c|c|c|c|}
\hline Variables & $\begin{array}{l}\text { Total sample } \\
(n=101)^{\star}\end{array}$ & $\begin{array}{l}\text { Intervention } \\
\text { Group } \\
(n=50)^{\star}\end{array}$ & $\begin{array}{l}\text { Control } \\
\text { Group } \\
(n=51)^{*}\end{array}$ & $P$ value \\
\hline \multicolumn{5}{|l|}{ Age } \\
\hline Mean (SD) & $70.1(6.8)$ & $69.7(6.0)$ & $70.5(7.5)$ & 0.52 \\
\hline Median (IQR) & $69(10.5)$ & $69.5(9.25)$ & $69(12.0)$ & 0.91 \\
\hline \multicolumn{5}{|l|}{ Type of UI, $n$ (\%) } \\
\hline Stress & $12(11.9)$ & $9(18.0)$ & $3(5.9)$ & 0.12 \\
\hline Urge & 37 (36.6) & $19(38.0)$ & $18(35.3)$ & \\
\hline Mixed & $52(51.5)$ & $22(44.0)$ & $30(58.8)$ & \\
\hline Duration of UI, median (IQR) & $9(15.75)$ & $8.5(11.5)$ & $10(16.0)$ & 0.94 \\
\hline Severity of UI, median (IQR) & $4(5.0)$ & $4(5.0)$ & $4(5.0)$ & 0.98 \\
\hline Body Mass Index, median (IQR) & $28.5(7.8)$ & $28.2(10.0)$ & $29.3(6.9)$ & 0.83 \\
\hline Hip/Waist, median (IQR) & $0.84(0.09)$ & $0.84(0.12)$ & $0.84(0.06)$ & 0.40 \\
\hline \multicolumn{5}{|l|}{ Co-morbidities } \\
\hline Hypertension, n (\%) & $47(46.5)$ & $26(52.0)$ & $21(41.2)$ & 0.28 \\
\hline Coronary Heart Disease, n (\%) & $20(19.8)$ & $8(16.0)$ & $12(23.5)$ & 0.34 \\
\hline Diabetes, n (\%) & $9(8.9)$ & $3(6.0)$ & $6(11.8)$ & 0.31 \\
\hline Chronic Airway Limitation, n (\%) & $13(12.9)$ & $8(16.0)$ & $5(9.8)$ & 0.35 \\
\hline Neurological condition, n (\%) & $18(18.0)$ & $10(20.0)$ & $8(16.0)$ & 0.63 \\
\hline
\end{tabular}

outcome variables between the treatment and control groups. The Wearer's diary data were coded, and reasons for use or non-use were categorized.

\section{Results}

Recruitment and randomization processes employed in the study worked well. Figure 2 demonstrates randomization and loss to follow-up. More than the originally planned 53 participants were recruited to each group to allow for loss to follow-up or withdrawal from the study. There was a high loss to follow-up associated with this study, and the final analysis of some of the secondary outcome variables included responses from less than 40 participants.

The main results of this study reports an intention-to-treat analysis that compares the outcomes of the treatment and control groups. As all outcome measures were self-reported and required the active participation of the subject, any subjects who withdrew from the study within 4 weeks of randomization or did not return data related to outcome measures were not included in the study. Of the 122 who were randomized to receive a treatment or placebo, only 101 provided some type of follow-up data $(82.8 \%)$. Tables 1 through 3 present the sample characteristics and baseline scores for the outcome measures.

There were no statistically significant differences in demographic characteristics, clinical characteristics, or baseline outcome measures between the intervention $(n=50)$ and the placebo $(n=51)$ groups. Comparison of demographic and clinical characteristics of those randomized but not included in the analysis $(\mathrm{n}=21)$ with those randomized and included in the analysis (figure 2) indicated no statistically significant differences between the groups.

Table 4 presents a comparison of changes in the outcome measures between baseline and 12 weeks for the treatment and control groups. None of the differences in changes in outcome measures between the groups from baseline to 12 weeks reached statistical significance.

At the end of data collection all participants were telephoned and asked whether they subjectively felt there had been an improvement in their urinary incontinence. In the intervention group, $56.5 \%(\mathrm{n}=46)$ felt there had been an improvement compared to $36 \%(\mathrm{n}=50)$ in the control group (chi square $=4.06$; $P=0.04)$.

A sensitivity analysis was conducted to determine whether the results were being influenced by a placebo effect. Those participants who correctly identified their group allocation were removed, and the comparison of the change in outcomes was undertaken again. This analysis revealed no statistically significant differences between the intervention and control groups for change in all the outcome measures between baseline and 4 weeks or baseline and 12 weeks.

The data were further analyzed to determine whether the results were being influenced by the degree to which participants adhered to the study protocol. Sixty-seven subjects $(66.3 \%)$ adhered to the study protocol to the level of $85 \%$, and $33(32.7 \%)$ adhered to the protocol $100 \%$. There was no difference in level of adherence between the groups. For the subjects that adhered to the protocol $85 \%$ of the time, the intervention group had better outcomes in the change in 
Table 2. Comparison of obstetric, gynecological and surgical history for intervention and control groups with descriptive statistics for total sample and groups.

\begin{tabular}{|c|c|c|c|c|}
\hline Variables & $\begin{array}{l}\text { Total } \\
\text { sample } \\
(n=101)^{\star}\end{array}$ & $\begin{array}{l}\text { Intervention } \\
\text { Group } \\
(n=50)^{\star}\end{array}$ & $\begin{array}{l}\text { Control } \\
\text { Group } \\
(n=51)^{\star}\end{array}$ & $P$ value \\
\hline \multicolumn{5}{|c|}{ Obstetric and gynecological history, $\mathrm{n}(\%)$} \\
\hline \multicolumn{5}{|c|}{ Vaginal deliveries } \\
\hline 0 & $8(7.9)$ & $5(10.0)$ & $3(5.9)$ & 0.49 \\
\hline 1 & $9(9.1)$ & $3(6.0)$ & $6(11.8)$ & \\
\hline 2 & $24(23.7)$ & $10(20.0)$ & $14(27.4)$ & \\
\hline $3+$ & $60(59.3)$ & $32(64.0)$ & $28(54.9)$ & \\
\hline \multicolumn{5}{|l|}{ Caesarean sections } \\
\hline 0 & 97 (96.0) & $48(96.0)$ & 49 (96.0) & 0.51 \\
\hline 1 & $3(3.0)$ & $2(4.0)$ & $1(2.0)$ & \\
\hline 2 & $1(1.0)$ & 0 & $1(2.0)$ & \\
\hline Perineal Tear/ Episiotomy & $69(68.3)$ & $36(73.5)$ & $33(66.0)$ & 0.68 \\
\hline Child $\geq 3.5 \mathrm{kgs}$ & $55(54.5)$ & $30(61.2)$ & $25(50.0)$ & 0.50 \\
\hline \multicolumn{5}{|l|}{ Continence related surgery, $\mathrm{n}(\%)$} \\
\hline Hysterectomy & $45(44.6)$ & $24(48.0)$ & $21(41.2)$ & 0.49 \\
\hline Pelvic Floor Repair & $17(16.8)$ & $9(18.4)$ & $8(15.7)$ & 0.72 \\
\hline Bladder Repair & $28(27.7)$ & $15(30.6)$ & $13(25.5)$ & 0.57 \\
\hline
\end{tabular}

Bothersomeness score at 12 weeks (Mann-Whitney $U=364.5$; $P=0.02$ ). No other between group differences reached statistical significance.

Qualitative data recorded by 96 of the 122 participants in the Wearer's Diary revealed problems with the comfort and wearability of the garment. Those wearing the intervention device also described difficulties related to the attraction of metal objects and the "magnets sticking to each other and making them difficult to put on".

\section{Discussion}

The results of the study reported in this article indicate that the sample was similar to those reported in other studies of older, community dwelling women with urinary incontinence. ${ }^{6}$ There were no differences between intervention and control groups at 3 months. On the most subjective measure (ie, when participants were asked over the telephone, at the end of the study, whether their UI had improved), the intervention group had a larger proportion that had experienced improvement compared to the control group, and this result reached statistical significance. These findings are similar to those reported in other smaller studies of electromagnetic and static magnetic stimulation using self-reported measures. ${ }^{9-10,13-14,17-18,27,33}$

The remainder of the self-reported measures and the objective measure of the Pad Test did not reveal any differences

Table 3. Outcome measures at baseline.

\begin{tabular}{llll}
\hline Variables & $\begin{array}{l}\text { Intervention } \\
(\mathbf{n = 5 0 )}, \\
\text { median (IQR) }\end{array}$ & $\begin{array}{l}\text { Control } \\
(\mathbf{n = 5 1 )}, \\
\text { median (IQR) }\end{array}$ & $\boldsymbol{P}$ value \\
\hline Incontinent episodes in 24 hr & $5(5.25)$ & $3.5(4.25)$ & 0.30 \\
Severity Index & $4(5.0)$ & $4(5.0)$ & 0.98 \\
Pad Test (g) & $36(96.75)$ & $20(83.0)$ & 0.40 \\
Bothersome scale & $8(4.0)$ & $7(5.0)$ & 0.86 \\
BFLUTS-FS (Filling) & $6(4.0)$ & $6(2.0)$ & 0.81 \\
BFLUTS-VS (Voiding) & $2(3.0)$ & $1(3.0)$ & 0.47 \\
BFLUTS-IS (Incontinence) & $10(4.0)$ & $10(4.25)$ & 0.57 \\
BFLUTS-sexual symptoms & $1(3.0)$ & $0.5(2.0)$ & 0.79 \\
BFLUTS-quality of life & $5.5(6.0)$ & $6(4.0)$ & 0.35 \\
\hline
\end{tabular}

BFLUTS, Bristol Female Lower Urinary Tract Symptoms; IQR, interquartile range 
Table 4. Comparison of treatment and control groups: Change in outcome measures - baseline to 12 weeks.

\begin{tabular}{|c|c|c|c|}
\hline Variables & $\begin{array}{l}\text { Intervention Group } \\
(\mathrm{n}=50),{ }^{*} \\
\text { median (IQR) }\end{array}$ & $\begin{array}{l}\text { Control Group } \\
(n=51),{ }^{*} \\
\text { median (IQR) }\end{array}$ & $P$ value $^{\dagger}$ \\
\hline Change in Pad Test (T1-T2) & $13.5(50.3)$ & $1.0(34.0)$ & 0.09 \\
\hline Change in frequency of incontinent episodes (T1-T2) & $0.75(2.63)$ & $0.5(2.0)$ & 0.68 \\
\hline Change in frequency of continent episodes (T1-T2) & $0.5(3.13)$ & $0.5(2.5)$ & 0.87 \\
\hline Change in Severity Index (T1-T2) & $1.0(3.0)$ & $0(2.0)$ & 0.59 \\
\hline Change in Bothersome scale (T1-T2) & $1.0(3.25)$ & $1.0(2.0)$ & 0.21 \\
\hline Change in BFLUTS - (UI symptoms) (T1-T2) & $1.5(3.0)$ & $1.5(3.0)$ & 0.80 \\
\hline Change in BFLUTS - (Filling) (T1-T2) & $1.0(2.0)$ & $1.0(2.0)$ & 0.51 \\
\hline Change in BFLUTS - (Voiding) (T1-T2) & $0(1.0)$ & $0(2.0)$ & 0.07 \\
\hline Change in BFLUTS- (Sexual Function) (T1-T2) & $0(0.75)$ & $0(0)$ & 0.80 \\
\hline Change in BFLUTS- (Quality of life) (T1-T2) & $1.0(5.0)$ & $0(5.0)$ & 0.28 \\
\hline
\end{tabular}

${ }^{*} n$ value is slightly less across some variables due to missing data.

${ }^{\dagger} P$ values derived from Mann Whitney test.

T1, Baseline; T2, 12 weeks; BFLUTS, Bristol Female Lower Urinary Tract Symptoms; UI, urinary incontinence; IQR, interquartile range

between the groups that reached statistical significance. Studies of electrical, electromagnetic, and static magnetic stimulation of the pelvis for the treatment of UI have been criticized for their small sample size. ${ }^{33}$ This study had based its power analysis on data from a self-reported study conducted by a manufacturer, and it seems that this data may have been optimistic. If there is an effect, it is considerably less than the $50 \%$ estimated by the manufacturer.

The sensitivity analysis, which included only those participants who could not identify their group allocation, indicated that when those who were not blinded to group allocation were removed from the analysis, the effect of the magnetic intervention was no longer apparent. This finding suggests that there might have been a placebo effect, and that subjectively, if people with UI believe that magnets will improve their condition, they may well experience improvement, but this improvement was not indicated on any of the more objective measures. This finding is not surprising since it has long been known that a significant placebo effect occurs in up to one-third of subjects, ${ }^{34}$ especially in the treatment of symptoms. ${ }^{35}$

Another factor that may have affected the outcome of this study, by contributing to poor protocol adherence, was the comfort of the garment. The data from the Wearer's Diary suggests that there are considerable design issues with the product, especially for use in the sub-tropical climate of South East Queensland. The garment is bulky and when worn over other undergarments and pads can be very warm to wear.

Although not all the participants who withdrew after consent provided reasons, the high early attrition rate of about $20 \%$ may be attributed to study participants deciding that the garment was not comfortable or appealing. As urinary incontinence is a condition which many women are careful to keep private, ${ }^{36}$ wearing a garment that could be visible under clothing or made obvious by its attraction to metal objects, may prove unattractive to many women. A certain level of manual dexterity is also required to put on and remove the undergarment, especially when in a hurry. The magnets are strong and require some adroitness to pull apart. For older women this was often a problem.

\section{Conclusions}

The study was well designed and conducted, although there are acknowledged difficulties in blinding participants in trials that involve magnets. The lack of statistically significant differences between the two groups could be due to various reasons: no magnetic effect on these outcomes, small sample size, issues related to design, and protocol adherence. The data from the Wearer's Diary suggested that there were some design issues with the product, especially for use in a subtropical climate. This meant that recruitment to a clinical trial was difficult, and adherence to the protocol was even more difficult. For these reasons, the lack of effect seen in the intervention group must be treated with caution. It may be that there could be an effect if the garment is worn as designed.

The effectiveness of the magnetic stimulation of the pelvis using static magnets incorporated into an undergarment cannot be determined from the results of this study. It is recommended that further work on garment design be undertaken to decrease the bulk and discomfort of the product. Further research needs to be undertaken into the basic physics of the strength and flux of the magnetic field generated by the product. Once this work is completed additional animal and clinical trials may be planned. Future 
clinical trials should have narrower inclusion criteria with respect to type and severity of UI and would need to include much larger sample sizes to ensure that the data analysis is sufficiently powered. Additionally, there is a need for a robust outcome measure to be used to determine sample size. Finally, creative strategies would have to be employed to ensure blinding of participants to group allocation.

\section{References}

1. Haylen BT, de Ridder D, Freeman RM, Swift SE, Berghmans B, Lee J, Monga A, Petri E, Rizk DE, Sand PK, Schaer GN, An International Urogynecological Association (IUGA)/ International Continence Society (ICS) joint report on the terminology for female pelvic floor dysfunction. Neurourol Urodyn 2010;29:4-20.

2. Hannestad YS, Rortveit G, Sandvik H, Hunskaar S: Norwegian EPINCONT study. Epidemiology of Incontinence in the County of Nord-Trøndelag. A community-based epidemiological survey of female urinary incontinence: the Norwegian EPINCONT study. Epidemiology of Incontinence in the County of Nord-Trøndelag. J Clin Epidemiol 2000;53:1150-1157.

3. Hunskaar S, Lose G, Sykes D, Voss S. The prevalence of urinary incontinence in women in four European countries. BJU Int 2004;93:324-330.

4. Ko Y, Lin S-J, Salmon JW, Bron MS. The impact of urinary incontinence on quality of life of the elderly. Am J Manag Care 2005;11:S103-S111.

5. Riss P, Kargl J. Quality of life and urinary incontinence in women. Maturitas 2011;68:137-142.

6. St John W, Wallis M. Outcome evaluation of a multi-disciplinary community-based continence service for Australian women. Women Health 2004;40:35-52.

7. St John W, Wallis M, Griffiths S, McKenzie S. Daily-living management of urinary incontinence: a synthesis of the literature. J Wound Ostomy Continence Nurs 2010;37:80-90.

8. Almeida FG, Bruschini H, Srougi M. Urodynamic and clinical evaluation of 91 female patients with urinary incontinence treated with perineal magnetic stimulation: 1-year followup. J Urol 2004;171:1571-1574; discussion 1574-1575.

9. Galloway NT, El-Galley RE, Sand PK, Appell RA, Russell HW, Carlan SJ. Extracorporeal magnetic innervation therapy for stress urinary incontinence. Urology 1999;53:1108-1111.

10. Finegold L, Flamm BL. Magnet therapy. BMJ 2006;332:4.

11. Galloway NT, El-Galley RE, Sand PK, Appell RA, Russell HW, Carlin SJ. Update on extracorporeal magnetic innervation (EXMI) therapy for stress urinary incontinence. Urology 2000;56:82-86.

12. Carlan SJ, Russell H, Shelnutt J, Peppy T, Griggs B. Extracorporeal magnetic innervation (EXMI) therapy in the treatment of urinary incontinence in women: results from a single center. J Am Geriatr Soc 2000;48:456.

13. Fujishiro T, Enomoto H, Ugawa Y, Takahashi S, Ueno S, Kitamura T. Magnetic stimulation of the sacral roots for the treatment of stress incontinence: an investigational study and placebo controlled trial. J Urol 2000;164:1277-1279.

14. Yamanishi T, Yasuda K, Suda S, Ishikawa N, Sakakibara R, Hattori T. Effect of functional continuous magnetic stimulation for urinary incontinence. J Urol 2000;163:456459.

15. Choe JH, Choo M-S, Lee K-S. Symptom change in women with overactive bladder after extracorporeal magnetic stimulation: a prospective trial. Int Urogynecol J Pelvic Floor Dysfunct 2007; 18:875-880
16. O’Reilly B, Fynes M, Achtari C, Hiscock R, Thomas E, Murray C, Dwyer PL. A prospective randomised doubleblind controlled trial evaluating the effect of trans-sacral magnetic stimulation in women with overactive bladder. Int Urogynecol J Pelvic Floor Dysfunct 2008;19:497-502

17. Gilling PJ, Wilson LC, Westenberg AM, McAllister WJ, Kennett KM, Frampton CM, Bell DF, Wrigley PM, Fraundorfer MR. A double-blind randomized controlled trial of electromagnetic stimulation of the pelvic floor vs sham therapy in the treatment of women with stress urinary incontinence. BJU Int 2009;103:1386-1390.

18. O’ Reilly B, Fynes M, Achtari C, Hiscock R, Thomas E, Murray C, Dwyer PL. A prospective randomised doubleblind controlled trial evaluating the effect of trans-sacral magnetic stimulation in women with overactive bladder. Int Urogynecol J Pelvic Floor Dysfunct 2008;19:497-502.

19. Hoșcan MB, Dilmen C, Perk H, Soyupek S, Armağan A, Tükel $\mathrm{O}$, Ekinci M. Extracorporeal magnetic innervation for the treatment of stress urinary incontinence: results of two-year follow-up. Urol Int 2008;81:167-172.

20. Doğanay M, Kilic S, Yilmaz N. Long-term effects of extracorporeal magnetic innervations in the treatment of women with urinary incontinence: results of 3-year followup. Arch Gynecol Obstet 2010;282:49-53

21. World Health Organization. Environmental Health Criteria 69: Magnetic fields. Geneva, Switzerland: World Health Organization; 1987.

22. Gmitrov J, Ohkubo C, Okano H. Effect of 0.25 T static magnetic field on microcirculation in rabbits. Bioelectromagnetics 2002;23:224-229.

23. Pittler MH, Brown EM, Ernst E. Static magnets for reducing pain: systematic review and meta-analysis of randomized trials. CMAJ 2007;177:736-742.

24. Colbert AP, Wahbeh H, Harling N, Connelly E, Schiffke HC, Forsten C, Gregory WL, Markov MS, Souder JJ, Elmer P, King V. Static magnetic field therapy: a critical review of treatment parameters. Evid Based Complement Alternat Med 2009;6:133-139.

25. Bergman J, Robertson JR, Elia G. Effects of a magnetic field on pelvic floor muscle function in women with stress urinary incontinence. Altern Ther Health Med 2004;10:70-72.

26. Thornton MJ, Kennedy ML, Lubowski DZ. Extracorporeal magnetic stimulation of the pelvic floor: impact on anorectal function and physiology. A pilot study. Dis Colon Rectum 2005;48:1945-1950.

27. Cohen J. Statistical power analysis for the behavior sciences. 2nd ed. Hillsdale, NJ: Routledge Academic; 1988.

28. Flisser AJ, Figueroa J, Bleustein CB, Panagopoulos G, Blaivas JG. Pad test by mail for home evaluation of urinary incontinence. Neurourol Urodyn 2004;23:127-129.

29. Thomas S, Moore K, Nay R, Fonda D, Marosszeky N. Continence Outcomes Measurement Suite: Project Final Report. Melbourne: LaTrobe University Faculty of Health Sciences; 2003. Available at: http:/www.health.gov.au/ internet/main/publishing.nsf/Content/E63B64D0F6C54D78C A257210007E5341/\$File/COMS\%20Screenr\%202006.pdf.

30. Brookes ST, Donovan JL, Wright M, Jackson S, Abrams P. A scored form of the Bristol Female Lower Urinary Tract Symptoms questionnaire: data from a randomized controlled trial of surgery for women with stress incontinence. Am J Obstet Gynecol 2004;191:73-82.

31. Sandvik H, Hunskaar S, Seim A, Hermstad R, Vanvik A, Bratt $H$. Validation of a severity index in female urinary incontinence and its implementation in an epidemiological survey. J Epidemiol Community Health 1993;47:497-499.

32. Stach-Lempinen B, Kirkinen P, Laippala P, Metsänoja R, Kujansuu E. Do objective urodynamic or clinical findings determine impact of urinary incontinence or its treatment on quality of life? Urology 2004;63:67-71; discussion 71-72. 
33. Shafik A. Re: Magnetic stimulation of the sacral roots for the treatment of urinary frequency and urge incontinence: an investigational study and placebo controlled trial. J Urol 2003;169:1800-1801.

34. Beecher HK. The powerful placebo. J Am Med Assoc 1955;159:1602-1606.

35. Cleophas TJ. The importance of placebo effects. JAMA 1995;273:283; author reply 284.

36. Fultz NH, Burgio K, Diokno AC, Kinchen KS, Obenchain R, Bump RC. Burden of stress urinary incontinence for community-dwelling women. Am J Obstet Gynecol 2003;189:1275-1282.

\section{Author Affiliations}

Marianne C. Wallis, RN, PhD ${ }^{*+}$; Elizabeth A. Davies, RN, PhD;

Lukman Thalib, PhDs; and Susan Griffiths, BA*

*Griffith University, Research Centre for Clinical and Community Practice Innovation, Queensland, Australia

The Gold Coast Health Service District, Queensland, Australia

The University of Queensland, School of Nursing, Queensland, Australia

sFaculty of Medicine, Kuwait University, Kuwait 